\title{
La formation des représentations relatives à l'addiction aux jeux d'argent : une comparaison entre la Chine et la France
}

The Formation of Representations relating to Gambling Addiction: a Comparison between China and France

Thomas Amadieu

\section{OpenEdition}

\section{Journals}

Édition électronique

URL : http://journals.openedition.org/sdj/2453

DOI : $10.4000 /$ sdj.2453

ISSN : 2269-2657

Éditeur

Laboratoire EXPERICE - Centre de Recherche Interuniversitaire Expérience Ressources Culturelles Education

\section{Référence électronique}

Thomas Amadieu, « La formation des représentations relatives à l'addiction aux jeux d'argent : une comparaison entre la Chine et la France », Sciences du jeu [En ligne], 13| 2020, mis en ligne le 05 novembre 2020, consulté le 21 janvier 2021. URL : http://journals.openedition.org/sdj/2453 ; DOI : https://doi.org/10.4000/sdj.2453

Ce document a été généré automatiquement le 21 janvier 2021.

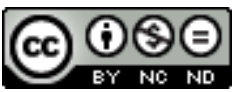

La revue Sciences du jeu est mise à disposition selon les termes de la Licence Creative Commons Attribution - Pas d'Utilisation Commerciale - Pas de Modification 4.0 International. 


\title{
La formation des représentations relatives à l'addiction aux jeux d'argent : une comparaison entre la Chine et la France
}

\author{
The Formation of Representations relating to Gambling Addiction: a Comparison \\ between China and France
}

Thomas Amadieu

1 Le modèle de l'addiction au jeu comme pathologie individuelle et son corollaire en matière d'auto-régulation de l'industrie, le «jeu responsable» (Blaszczynski, Ladouceur \& Shaffer, 2004), semblent devoir s'imposer dans un nombre croissant de pays en même temps que l'offre de jeux s'ouvre aux marchés; ils s'imposent pour de bonnes raisons, notamment car ils rendent des services intellectuels et pratiques aux législateurs et aux opérateurs. Le modèle du jeu responsable propose une forme de compromis aux régulateurs publics en les déchargeant de la responsabilité de fournir un cadre réglementaire contraignant au profit d'actions volontaires de l'industrie du jeu, tout en offrant une assise scientifique partielle à ces dernières (Hancock et Smith, 2017).

2 Avec le développement des marchés des jeux d'argent (jeux en ligne, paris sportifs et hippiques, poker et jeux de carte, jeux de tirage et de grattage, machines à sous, etc.), on aurait pu envisager une diffusion de ce modèle à mesure que les opérateurs privés doivent communiquer sur leur éthique auprès de consommateurs soucieux de leur santé. Mais l'extension du marché mondial des jeux d'argent et sa libéralisation avancée ne paraissent pas se traduire par une convergence complète des régimes de régulation et des représentations de la pratique. En témoignent les deux modèles proposés ici à la comparaison : le modèle libéral français et le modèle restrictif chinois. Le modèle libéral (ou "modèle du risque ») repose sur la science comme source de légitimité, car elle permet la quantification du risque et fournit une justification au jeu comme activité de loisir (Kingma, 2008). À l'inverse le modèle restrictif chinois (ou 
«modèle de l'alibi ») considère le jeu comme une activité foncièrement condamnable moralement, tout en tolérant une offre de jeu (parfois de façon spectaculaire dans le cas de Macao) qui se veut canalisée au service du bien commun.

3 Ainsi la médicalisation, c'est-à-dire le "processus par lequel des problèmes nonmédicaux sont progressivement définis et traités comme des problèmes médicaux " (Conrad, 1992 ; p. 209) ne s'opère pas de façon uniforme. Faut-il voir dans l'absence relative de médicalisation du jeu en Chine un «retard» dans une tendance qui constituerait un progrès des connaissances ? L'hypothèse de cet article est au contraire d'explorer la possibilité que l'adoption du modèle du jeu responsable repose sur un ensemble de représentations culturellement situées ainsi que des contextes institutionnels spécifiques. En prenant appui sur une comparaison avec la Chine, l'article souligne l'imperméabilité relative de la société chinoise au modèle médical anglo-saxon, associé à des régimes de plus grande liberté individuelle. La Chine et la France ont des usages ludiques et des représentations du jeu différents, basés sur des déterminants historiques et socio-politiques distincts, qui mènent à des conceptualisations des problèmes de jeu et à des modalités d'intervention-prévention dissemblables.

4 La médicalisation du jeu tend à imposer une représentation nouvelle du jeu dont il faudra retracer la généalogie pour mieux saisir ce qui fait obstacle à son déploiement. La représentation sociale est ici appréhendée comme un ensemble de connaissances et croyances partagées participant de la construction d'une réalité commune (Jodelet, 2003). Là où le discours médical en France offre pour partie une déresponsabilisation à l'individu qui est à soigner, en Chine la condamnation morale perdure en tant que le joueur met en danger son entourage, sa famille et plus largement l'harmonie sociale (Hu, 2015). Alors que dans le régime de représentation libéral le joueur dépendant devient victime, dans la représentation morale chinoise celui-ci demeure coupable $(\mathrm{Wu}$ et Lau, 2015).

Pour répondre à cette interrogation, une comparaison sera dressée à différents niveaux de représentations clés: le niveau scientifique, le niveau opérationnel politicoindustriel et enfin le niveau des représentations ordinaires. La comparaison avec le terrain chinois est intéressante à plus d'un titre et je voudrais montrer sa portée heuristique. L'idée n'est pas d'adopter une perspective culturaliste afin de contempler des différences radicales, mais de chercher à préciser les contours et mécanismes contribuant à transformer les représentations du jeu.

\section{Un processus de médicalisation faillible?}

\section{En France, un processus de médicalisation importé tardivement}

6 Le processus de médicalisation, comme Amnon Suissa le mentionne dans un article de 2005, conduit à ce que « ce qui était auparavant considéré comme un péché, un vice, un comportement déviant et une industrie hors-la-loi est aujourd'hui compris comme une maladie, une pathologie psychiatrique teintée d'une perte de contrôle ou d'un désordre d'impulsion. » (Suissa, 2005, p. 11). Les travaux sur la médicalisation du jeu, désormais bien documentés (Rosecrance, 1985; Rossol, 2001; Campbell \& Smith, 2003; Reith, 2007; Cosgrave, 2008), reprennent notamment la théorie de la médicalisation développée d'abord par Conrad $(1980,1992,2005)$, qui révèle le rôle moteur des 
médecins dans la médicalisation de l'hyperactivité ou la ménopause par exemple, ainsi que le rôle d'associations telles que les Alcooliques Anonymes dans la médicalisation de l'alcoolisme, contre les résistances et hésitations initiales des médecins. Les agents moteurs de la médicalisation pouvant varier selon les pathologies et les contextes, aussi le jeu problématique a-t-il ses propres prescripteurs de normes.

7 Rosecrance reprend dès 1985 ce modèle pour montrer que la notion de jeu pathologique procèderait d'un travail d'étiquetage, dans une logique de domination politique, de la part de groupes d'entraide ou thérapeutique et de cliniciens pour faire inscrire le jeu comme pathologie, en accord avec les représentations des «classes moyennes ». Cette perspective critique part du postulat qu'à l'état naturel, il n'existe pas de preuve empirique de l'existence de la maladie et que la médicalisation n'est qu'un effort de contrôle. L'inspiration de ces travaux est clairement foucaldienne, même s'il n'est pas cité : l'étiquetage de la maladie embrasserait la pente du sens commun pour exercer une forme de domination en stigmatisant une déviance (Rosecrance, 1985). La médicalisation comme forme de contrôle social peut être questionnée, dans la mesure où les régimes libéraux ne semblent pas percevoir un quelconque danger dans le comportement de jeu, même excessif; au contraire le jeu assure des ressources confortables aux opérateurs publics ou à l'État via les ressources fiscales. On pourrait à l'inverse se demander si le processus de médicalisation ne procèderait pas des actions d'une pluralité d'acteurs ayant des intérêts convergents.

Ces travaux se situant dans le contexte anglo-saxon, ils soulignent l'influence d'entrepreneurs de morale (Becker, 1963), souvent des groupes d'entraide d'inspiration religieuse. Ce modèle de processus pourrait différer lorsqu'il est transposé dans d'autres contextes culturels. L'exemple de la France est à cet égard éclairant: on constate un « retard» important, dans la mesure où de tels groupes, s'ils existent ont une influence réduite à la fois sur les représentations ordinaires et sur les pouvoirs publics eux-mêmes. En France, on observe peu de pression ascendante (bottom-up) pour médicaliser le jeu émanant d'associations, qu'elles soient religieuses ou non. Néanmoins, dans la période récente, sous l'impulsion de certains opérateurs et d'organismes parapublics, donc clairement dans un sens descendant (top-down), une médicalisation tardive s'opère; l'enjeu étant notamment de fournir des garanties au niveau européen au moment de l'ouverture à la concurrence des jeux en ligne en 2010.

La facilité avec laquelle le modèle peut s'imposer tient au fait d'abord que l'assemblage conceptuel est prêt à l'importation : une classification (DSM, Manuel diagnostique et statistique des troubles mentaux, dans ses versions successives, American Psychiatric Association, 2013), des diagnostics (le South Oaks Gambling Screen - SOGS - et l'Indice canadien du jeu excessif - ICJE), des modèles de prise en charge et des outils RSE (Responsabilité Sociale des Entreprises) pour les opérateurs. En France, le «jeu responsable " est la forme privilégiée de mise en pratique du modèle savant biopsychologique par les opérateurs de jeu; il importe des pays anglo-saxons le modèle formalisé à l'origine sous le nom de "modèle de Reno " par Blaszczynski, Ladouceur \& Shaffer (2004). Il s'agit d'un ensemble de «bonnes pratiques » visant à prévenir ainsi qu'à réduire les problèmes de jeu par l'information aux risques auprès des joueurs et des employés, des dispositifs de contrainte et d'auto-contrainte (Bonello \& Griffiths, 2017 ; Hing, Russell, \& Hronis, 2018). 
10 Ce modèle médical impose son propre découpage du réel, ses catégories nosographiques qui distinguent le normal du pathologique, comme le souligne Brody (2015) :

Si la frontière entre le normal et le pathologique renvoie sans doute à une réalité clinique ou empirique, elle n'en demeure pas moins fondée sur des normes, au même titre que la frontière entre la raison et la passion, le vice et la vertu, le bien et le mal. Le problème, c'est que ces normes - comme la plupart des normes sociales s'ignorent comme telles.

11 Pour une part, néanmoins, les concepteurs de ces manuels psychiatriques (American Psychiatric Association, 2013) cherchent aujourd'hui à renforcer l'aspect de spectre, en adoptant des nosographies organisées en continuums censés permettre d'avoir une image plus fidèle du réel. Reste qu'au niveau opérationnel, les manuels de diagnostics doivent permettre d'arbitrer entre la pertinence d'un traitement ou la responsabilité d'une personne, donc in fine la frontière sera toujours tracée de façon dichotomique entre le normal et le pathologique, avec ce qu'elle a de contingente etde critiquable. Si c'est à ce prix que le scientifique peut avoir un impact sur le réel, ces catégories ouvrent toutefois la voie à des représentations unicausales et simplificatrices.

12 Pourtant le modèle médical n'est pas un bloc uniforme, il évolue, donne lieu à des controverses. Ainsi observe-t-on des évolutions dans les systèmes de classification, dont les versions successives donnent à voir les sédimentations de débats et des accords à un instant donné. Le jeu est reclassé de la catégorie des troubles du contrôle des impulsions ("impulse control disorder ») aux troubles addictifs (" addictive behavior») lors du passage du DSM-IV au DSM-5. Si les manuels reflètent une approche traditionnelle cherchant à établir des catégories discontinues permettant de mesurer des taux de prévalence et de poser des diagnostics, l'approche qui tend à être privilégiée désormais est celle d'une réduction des dommages causés, dans une logique de réduction des risques ( harm minimization») qui correspond à une approche plus complète en santé publique (Hunt et Blaszczynski, 2019).

On le voit, le rôle de la science, et tout particulièrement de la psychiatrie, est crucial dans le processus de médicalisation. Une forme de compétition s'est installée entre les sciences, notamment entre, d'une part, les sciences sociales qui poussent à questionner la dimension collective $\mathrm{du}$ jeu et, de l'autre, les sciences biopsychologiques qui envisagent essentiellement les mécanismes individuels. Il n'a pas été assez mentionné que les succès de la médicalisation sont peut-être liés à leur capacité à se conformer de façon supérieure aux canons de la science et de l'administration de la preuve (résultats cumulatifs, réplicabilité, méta-analyses, telles que celle de Dowling et al., 2017). Il est ainsi possible qu'au-delà des services rendus aux décideurs publics et à l'industrie, ils offrent une explication plus plausible et pouvant s'appuyer sur un ensemble de données convaincantes.

14 Dans cette compétition, les travaux reposant sur une approche biopsychologique individualisante semblent pouvoir bénéficier de financements par l'industrie plus importants, ce qui soulève des questions éthiques importantes et conduit à un manque de connaissances relativement aux causes sociales des problèmes associés au développement rapide de l'offre de jeux d'argent au cours des dernières années (Livingston \& Adams, 2016). L'instrumentalisation de la science par les industries aux fortes externalités négatives est un procédé déjà éprouvé, notamment par l'industrie du tabac. Le contrôle de l'agenda scientifique se fait par le biais de financements fléchés 
voire, dans certains cas, d'enquêtes falsifiées (Cassidy, 2014 ; Cowlishaw \& Thomas, 2018).

15 Mais au-delà de cette forme de compétition entre disciplines pour la reconnaissance institutionnelle et la prise en compte des résultats par les politiques publiques, il y a des particularités nationales au sein même d'une discipline.

\section{Une médicalisation possible en Chine?}

16 En Chine, le jeu d'un point de vue nosographique n'est toujours pas considéré comme une addiction mais comme un trouble compulsif, comme c'était le cas dans le DSM-IV. La Chine dispose de son propre système de classification alternatif au DSM, le CCMD 3 ou Classification Chinoise des Troubles Mentaux (Chinese Society of Psychiatry, 2003). C'est une différence de nature importante relativement à la prise en compte de la pathologie, qui s'accompagne d'une absence de prise en compte réelle des problèmes de jeu par les pouvoirs publics.

17 Les associations disciplinaires nationales, en l'occurrence celles de la psychiatrie, peuvent manifester des divergences comme dans le manuel qui constitue leur référence. Dans le monde, la CIM-11 (Classification Internationale des Maladies) produit par l'Organisation Mondiale de la Santé (World Health Organisation, 2018) est la référence, en même temps que le DSM-5 produit lui par l'Association Américaine de Psychiatrie (APA, 2013). Ces écrits nosologiques ont convergé pour se ressembler fortement, la plupart des associations de psychiatrie les utilisent, à l'exception notable donc de la Chine. Le DSM étant très américain (il repose principalement sur des études américaines), son alternative chinoise introduit des différences en matière de diagnostic censées être adaptées au contexte culturel, mais aussi politique et institutionnel (Lee, 2001).

18 En Chine, ayant longtemps été formés en Union soviétique, les psychiatres sont pour beaucoup méfiants vis-à-vis des influences américaines. L'influence politique est évidente et directe dans la construction du manuel chinois de psychiatrie (Lee, 2001). Plus largement, la Chine a une tradition médicale qui lui est propre. Bien sûr, elle dispose d'une médecine moderne, mais on observe des tentatives de faire cohabiter les deux et d'établir la spécificité de sa vision du monde. Très inspiré du manuel américain, celui chinois se distingue notablement par le maintien de l'homosexualité comme pathologie ou encore l'addition de la «psychose du voyage » («travelling psychosis») et $\mathrm{du}$ " trouble mental associé au Qigong " (" qigong-induced mental disorder ») et surtout, dans notre cas, la suppression du « jeu pathologique » comme catégorie à part entière et son classement avec les jeux vidéo dans la catégorie des « troubles de l'habitude et de l'impulsion " (" habit and impulse disorders »).

Des efforts sont toutefois faits pour adapter les critères de diagnostics internationaux et outils d'évaluation et de dépistage au contexte chinois, mais ils restent relativement marginaux en Chine continentale. Ces adaptations sont souvent élaborées par des chercheurs issus d'universités non-chinoises (Australie, Nouvelle-Zélande) et testés empiriquement sur des populations issues de la diaspora ou bien à Hong-Kong et Macao (Tao et al., 2011). Il est possible de mentionner, concernant le dépistage (screening) psychiatrique, l'élaboration d'une version chinoise du South Oaks Gambling Screen (le CSOGS) testée psychométriquement, notamment en regard de son équivalent étranger auprès de la population chinoise ${ }^{2}$. Ou plus largement les créations de questionnaires sur 
les attitudes face au jeu; ainsi plusieurs travaux, souvent parus dans le Journal of Gambling Studies, proposent des adaptations des échelles développées dans le contexte occidental en langue chinoise et à destination du public chinois: inventaire des motivations, attitudes et comportements de jeu (Tao et al., 2011), cognitions liées au jeu (Oei, Lin \& Raylu, 2007a ; Wu et Tang, 2011) ou encore besoins et désirs liés au jeu (Oei, Lin \& Raylu, 2007b).

Un nombre croissant de chercheurs chinois appellent de leurs vœux une reconnaissance $d u$ "jeu pathologique " ou des "troubles liés au jeu d'argent» (Gambling Disorder), notamment à Hong Kong et Macao. Certains chercheurs souhaitent une convergence de la prise en compte du jeu vers des standards internationaux ayant fait leurs études aux États-Unis ils sont très influencés par la sphère anglo-saxonne. Citons l'article de Wu et Lau (2015) promouvant le modèle médical du jeu responsable et invitant à une prise en compte des problèmes de jeu en Chine continentale, sous la forme d'un modèle de jeu responsable adapté au contexte chinois, qui impliquerait certaines spécificités comme la prise en compte des superstitions et de l'importance de la famille.

21 Mais une enquête de 2008 réalisée par questionnaire auprès de psychiatres chinois témoignait d'un usage privilégié du manuel chinois et d'une uniformisation/ internationalisation contrastée (Zou et al., 2008). Ainsi, à Pékin, sur 180 psychiatres, $64 \%$ utilisaient le manuel chinois, tandis que seuls $29 \%$ employaient la CIM-10 et $8 \%$ le DSM-IV. Une forte minorité considérait que les manuels étrangers étaient difficiles à adapter au terrain chinois pour des raisons de différences culturelles et qu'ils étaient « trop ancrés dans les concepts et les valeurs culturelles européennes » (Zou et al., 2008, p. 56). Ces données un peu anciennes témoignent toutefois de particularités nationales très fortes en matière de psychiatrie du jeu, une uniformisation des usages cliniques dans le sens d'une occidentalisation demeure très incertaine. Plus récemment, une enquête par questionnaire soulignait que, sur 110 psychiatres chinois interrogés, un seul avait reçu une formation consacrée aux problèmes liés aux jeux d'argent (Huang, Shijun \& George, 2014).

\section{Quelle influence des représentations savantes sur les représentations politiques?}

\section{Le modèle libéral du jeu responsable}

22 Historiquement prohibitionniste, la France est en transition avancée vers un régime libéral (Kingma, 2008) dans lequel le jeu d'argent est transformé en marchandise disponible sur un marché et devient une pratique de loisir légitime associée à une consommation aux risques limités. La privatisation de la Française des Jeux (FDJ) constitue ainsi le dernier signe de cette libéralisation, quelques années après l'ouverture à la concurrence des jeux en ligne. Les restrictions sont toutefois nombreuses, produit d'une histoire mouvementée et de rationalités ex post censées être protectrices des joueurs et de l'ordre public.

Ce régime de régulation des risques libéral français s'accompagne d'un effort de légitimation du marché face à la possible contestation associée aux coûts sociaux du jeu. La justification du marché des jeux prend la forme privilégiée du «jeu responsable " comme modèle d'auto-régulation de l'industrie, issu du «modèle de 
Reno » conçu par Blaszczynski, Ladouceur \& Shaffer (2004). Il consiste à responsabiliser le consommateur en même temps qu'à laisser aux opérateurs la responsabilité du financement des recherches et de la prévention face aux risques associés au jeu (Hancock \& Hao, 2016). Le modèle du jeu responsable repose sur la mise en place d'outils censés renforcer ou maintenir l'« agentivité » (capacité d'agir) des joueursconsommateurs.

Dans ce cadre, l'addiction est la modalité adoptée par les pouvoirs publics pour la gestion des externalités négatives du marché. Elle permet de laisser le marché s'étendre, tout en considérant qu'une minorité malade de joueurs doit être protégée d'elle-même et être pour partie prise en charge médicalement. Ainsi, en France, le modèle médical de l'addiction au jeu a acquis une place dominante dans le discours savant, pour différentes raisons dont il est possible de faire l'hypothèse, telles que les possibilités de financement de la recherche ou la capacité à publier des articles dans des revues de référence. Ce discours est repris et en retour encouragé à la fois par les industriels du jeu qui y ont intérêt dans le cadre de leurs politiques de RSE (Mangel et Trespeuch, 2009), ainsi que par les pouvoirs publics au sens large. Dans une enquête menée auprès de chercheurs du domaine des études sur les jeux d'argent (gambling studies), Cassidy, Loussouarn \& Pisac (2013) mettent au jour les mécanismes cognitifs par lesquels les relations financières avec l'industrie du jeu augmentent la probabilité de conclusions pro-industrie par les biais favorables et les sentiments de reconnaissance que ces relations suscitent (Cassidy, 2014).

Le «jeu responsable» est l'extension au domaine des jeux d'argent de catégories intellectuelles et d'outils pratiques appliqués par ailleurs dans le cadre des politiques dites de RSE. Les prémices françaises d'un engagement RSE appliqué aux jeux d'argent sont des initiatives isolées de certains casinos, à la fin des années 2000, afin de limiter les conséquences néfastes pour l'activité de joueurs rencontrant des problèmes de jeu. Ainsi le casino Barrière de Lille avait embauché une psychologue à temps plein dont la mission était d'informer les usagers et de gérer les joueurs présentant des signes de problèmes de jeu. D'autres opérateurs adoptent des pratiques similaires visant à sensibiliser les employés aux problèmes associés aux jeux, comme la FDJ qui se dote de moyens de promotion du «jeu responsable " avec un service dédié et une activité importante de financement de recherches sur la question (thèses en convention industrielle de formation par la recherche, dites CIFRE, partenariats universitaires, etc.). Ces mesures de prévention expérimentales et volontaires ont par la suite été rendues obligatoires en préparation de l'ouverture à la concurrence des jeux en ligne et afin que des garanties soient offertes en matière de protection des joueurs et de l'ordre public, les jeux étant une activité encore controversée. En France, le jeu responsable est donc le pendant logique de l'ouverture à la concurrence et de l'expansion de l'offre de jeu via la libération du marché.

26 Ces mesures de jeu responsable correspondent à une logique libérale de gouvernance morale du consommateur qui suppose la création active et le management d'un consommateur rendu responsable de ses actions (Giesler \& Veresiu, 2014). L'attirail de mesures proposé est destiné à renforcer l'agentivité du consommateur: il doit être majeur (pour faire un choix mature), être informé des risques qu'il prend en jouant (tels que des risques de dépendance ou de solitude), être informé de la possibilité de s'auto-exclure et de fixer des limites à sa propre consommation, être tenu informé également des sommes misées précédemment. Les postulats sur lesquels le paradigme 
repose s'apparentent à ceux de l'homo œconomicus des économistes néo-classiques (Demeulenaere, 1996). Semble ainsi ignoré le fait que la responsabilité individuelle peut être affectée par l'addiction ou des vulnérabilités individuelles - qu'elles soient d'origine sociale ou biopsychologiques. (Campbell \& Smith, 2003; Hancock, Schellinck \& Schrans, 2008; Hancock \& Smith, 2017; Livingstone \& Woolley, 2007; Schüll, 2012).

On peut donc s'interroger sur l'efficacité de ces mesures. Le niveau d'addiction au jeu n'a pas diminué en France depuis leur instauration, et aucune étude n'a établi de corrélation entre ces mesures et la diminution des problèmes associés au jeu. Les mesures de protection des joueurs peuvent être de type primaire, c'est-à-dire de prévention (Monaghan \& Blaszczynski, 2010) ou de type secondaires - plus répandues, elles ciblent alors les joueurs à risque à travers des auto-évaluations ou en fonction de leur consommation : ce sont les mesures d'auto-exclusion ou de fixation de limites. $\mathrm{Ce}$ type de mesure n'est efficace que lorsque les contrôles sont obligatoires (vérification d'identité par exemple). Il existe des algorithmes permettant de repérer les joueurs en ligne à risque en fonction de leurs comportements de jeu, mais ils sont rarement mis en œuvre au profit de mesures cosmétiques ou de messages d'information culpabilisants qui insistent sur la responsabilité des joueurs en dédouanant l'opérateur (Marionneau \& Järvinen-Tassopoulos, 2017). Dans leur enquête sur les sites de jeu et paris en ligne en France, Marionneau \& Järvinen-Tassopoulos (2017) soulignent que les mesures de jeu responsable adoptées prioritairement sont celles qui ne fonctionnent pas, tandis que les mesures efficientes qui permettraient de réellement repérer les joueurs "à risque " et de limiter l'offre pour les plus vulnérables sont ignorées (Wood \& Wohl, 2015 ; Harris \& Griffiths, 2017).

Ces différents aspects témoignent des capacités limitées du marché à s'autoréguler en matière de protection des joueurs. Ce modèle tend paradoxalement à déresponsabiliser les pouvoirs publics et les opérateurs. Il porte la lumière sur le seul phénomène de l'addiction au jeu (Problem Gambling), qui ne représente pourtant qu'un pourcentage limité des conséquences sociales négatives associées aux jeux d'argent (Browne et al., 2016). Sont ainsi éludés les problématiques associées à l'impact des jeux d'argent sur les classes populaires (Duroy \& Wisman, 2017).

\section{En Chine une vision morale assortie d'une politique de limitation de l'offre}

Après la révolution et l'accession des communistes au pouvoir en 1949, les jeux d'argent dans leur totalité ont été interdits, avant que des loteries ne soient introduites dans les années 1980, suivant l'ouverture économique. En principe, la Chine est prohibitionniste en matière de jeu, mais les exceptions sont nombreuses. Il demeure que la Chine, tout comme de nombreux pays éloignés de la sphère d'influence angloaméricaine, paraît prendre le contrepied du modèle libéral privilégié par la France : la tendance est au contrôle politique des comportements via une offre fortement canalisée et le plus souvent prohibée.

30 À la différence de la France, en Chine continentale, le concept de jeu responsable était jusqu'à une période récente totalement inexistant (Wu \& Lau, 2015). Si à Macao et Hong-Kong certaines mesures de protection peuvent être mises en place avec le soutien de l'industrie du jeu, elles restent peu appliquées et mal comprises, à la fois par les joueurs et les employés des casinos (Tong et al., 2018). Il n'existe pas de programme 
national de prise en compte des problèmes de jeu ni véritablement de services de soins ou d'entraide à destination des joueurs (Wu \& Lau, 2015).

31 Afin de canaliser l'offre de jeu et dans un but officiel de charité, l'État chinois autorise et organise deux loteries (caipiao - 彩票), la China Welfare Lottery et la China Sports Lottery. Ces loteries d'État, organisant des tirages aléatoires de nombres, ont généré depuis leur création des revenus toujours croissants, avec une augmentation annuelle en moyenne de 39,4\% depuis 1987, selon Chuancai Securities Co Ltd. ${ }^{3}$ En 2018, les revenus ont été estimés à plus de 500 milliards de Yuans (63,8 milliards d'euros environ) et, malgré un recul, en 2019 la popularité de ces loteries reste importante selon les chiffres compilés par la World Lottery Association qui recueille les données émanant des deux opérateurs chinois. La condition de l'existence des loteries d'État est la possibilité d'euphémiser la pratique: les loteries ne sont pas explicitement présentées comme des jeux d'argent (gambling), mais comme un projet social au bénéfice de l'État et de bonnes œuvres; celui qui participe à la loterie fait un acte de charité.

Récemment toutefois ont commencé à apparaitre des références au jeu responsable sur les sites institutionnels des deux loteries d'État, ${ }^{4}$ qui sont désormais certifiées par la World Lottery Association (au niveau 3 sur 4 niveaux de certification). En obtenant cette certification, les deux opérateurs ont dû en principe fixer un plan pour développer des politiques de jeu responsable et s'engagent à conduire ou financer des recherches sur le jeu responsable. Dans le domaine des jeux de hasard comme dans d'autres, la Chine cherche à se conformer ou prendre part à la construction de normes d'organisations de coopération et de régulation multilatérales. Cette instance étant financée par ses membres, elle participe d'une forme de légitimation internationale des opérateurs de jeu par une auto-évaluation n'offrant pas toutes les garanties d'indépendance.

Si certaines entreprises privées chinoises s'engagent par ailleurs dans le marché des jeux par des voies détournées, notamment par la conception de machines à sous, l'État n'est pas résolu à laisser proliférer l'offre de jeu sur le territoire national et aucune libéralisation ne paraît à l'ordre du jour. Lorsque l'on visite les points de vente, on est loin de l'exubérance des casinos, les espaces sont exigus et l'offre réduite. En 2019, des mesures ont été prises pour réduire l'attractivité des jeux, notamment en limitant les volumes des gains possibles ainsi que la fréquence des tirages.

34 La gouvernance du jeu en Chine demeure avant tout une politique de lutte contre le jeu illégal et le crime organisé. Cette volonté répressive est visible dans les échos portés par la presse officielle qui traite le jeu sous l'angle de la lutte contre la criminalité par l'État chinois. Malgré ces efforts, le jeu d'argent est extrêmement répandu en Chine, que ce soient les jeux de cartes ou le mahjong, mais aussi en ligne avec des moyens détournés d'accéder aux sites. Le crime organisé chinois tire d'importants revenus du jeu, en Chine et à l'étranger; le jeu constituant sa source de revenus principale en Chine continentale (Wang \& Antonopoulos, 2015). L'International Centre for Sport Security, un organisme basé au Qatar, a estimé en 2016 que les paris sportifs illégaux représentaient 600 milliards de dollars par an en Chine. Alors que l'État cherche officiellement à exercer un contrôle de plus en plus étroit sur les jeux illégaux, il n'y parvient donc pas complètement.

35 L'usage des téléphones intelligents ou smartphones s'étant généralisé en Chine (pas seulement dans la classe moyenne), de nombreux opérateurs basés à l'étranger (au 
Costa Rica et aux Philippines notamment) proposent des jeux destinés au public chinois (baccarat, blackjack, paris sportifs, loteries, etc.) avec une interface en chinois. Des centaines de millions de yuans échappent ainsi au contrôle de l'État chinois en partant vers l'étranger, en toute illégalité puisque le jeu - y compris en ligne - est interdit en Chine continentale. De plus, les Chinois sont nombreux à voyager pour jouer, notamment dans les pays limitrophes qui ciblent spécifiquement les joueurs Chinois (Cambodge, Philippines, Singapour). La préoccupation de l'État est avant tout de limiter la propagation du jeu, qui, s'il représente une manne fiscale, fait peser aussi des risques financiers et sociaux. Aussi malgré les bénéfices économiques que l'administration chinoise pourrait tirer d'une levée de la politique de prohibition, cette option ne fait pas l'objet de débats officiels.

En dépit d'une vraie volonté de contrôle social total de la part de l'État chinois, le modèle du jeu pathologique ne semble pas (encore?) s'imposer et la médicalisation parait susciter des réticences. Des initiatives spectaculaires de médicalisation peuvent ponctuellement apparaître, mais elles sont généralement par la suite désavouées par l'État tout en étant très éloignées du modèle du jeu responsable. Citons l'exemple bien connu du psychiatre Yang Yongxin en 2009 qui, après avoir reçu une bourse gouvernementale pour ses travaux, a été l'objet d'une vague d'indignation pour avoir utilisé des méthodes par "chocs thérapeutiques » électriques sans anesthésie sur des enfants ayant supposément une addiction à internet. ${ }^{5}$ En 2019, une nouvelle clinique pour l'addiction aux jeux a ouvert dans un grand hôpital de Pékin en suscitant un grand nombre de réactions négatives. Ainsi la gouvernementalité du jeu par la médicalisation et les politiques de jeu responsable ne paraissent pas s'imposer en Chine. Même à Macao, qui est sans doute la capitale mondiale du jeu, à la pointe du progrès technologique et marketing (Paulès, 2016), si la reconnaissance faciale est utilisée par les casinos pour identifier et cibler les joueurs les plus profitables ou les contrevenants, ces outils ne sont pas mobilisés pour l'imposition de normes de jeu responsable. Les réticences à l'adoption d'un mode de gouvernance morale du consommateur responsable trouvent peut-être leur origine dans les représentations sociales ordinaires.

\section{Les représentations ordinaires : vision morale contre vision médicale?}

\section{Le jeu excessif comme tare morale en chine}

37 Cette absence relative d'une véritable politique de jeu responsable en Chine fait écho aux représentations du jeu chez les Chinois : le jeu excessif est perçu comme une tare morale, pas comme une pathologie (Liu, 2009, 2010; Papineau, 2005). Les rapports à l'argent et au jeu ont évolué de façon spectaculaire en Chine. La société chinoise est passée de la période de collectivisation des moyens de production et de la Révolution Culturelle, de la valorisation du travail manuel (ouvrier et paysans) et d'un ascétisme/ puritanisme idéologiquement lié à l'édification du communisme, à une période d'ouverture économique durant laquelle les Chinois étaient officiellement encouragés à s'enrichir. mouvementée, le jeu sous ses différentes formes ayant été tantôt associé à un vice 
importé de l'Occident par des puissances étrangères coloniales malveillantes et corruptrices (les tripots et salles de jeu de Shanghai, associés à l'opium), tantôt comme une façon ingénieuse de lever un impôt pour les finances publiques (financement de grands travaux publics par des loteries). Aujourd'hui, après une période d'assouplissement, le contrôle plus étroit exercé sur les comportements individuels s'apparente à une forme d'ordre moral auquel chacun est invité à se plier, ce contrôle étant renforcé par la mise en place désormais fameuse du crédit social qui permet de sanctionner positivement ou négativement une série de comportements dont la liste n'est pas encore connue et peut varier d'une initiative à l'autre (Kostka, 2019; Ramadan, 2018). Ainsi les comportements d'achats peuvent être sanctionnés de limitations (acheter un billet d'avion ou de train) en cas de comportements déviants, par exemple lorsqu'une personne ne s'acquitte pas d'une dette. Plus directement, les pratiques de jeu sont visées, comme cela a été le cas dans le cadre de la lutte contre la corruption à Macao ou concernant les jeux vidéo qui doivent désormais respecter une charte stricte relative aux valeurs véhiculées. On observe donc une forme de retour à un contrôle social plus étroit, les Chinois n'étant plus seulement invités à prospérer, mais plutôt à faire prospérer leur pays.

Pour autant le jeu est très populaire en Chine et dans la diaspora (Loo, Raylu \& Oei, 2008), particulièrement chez les jeunes hommes pour des raisons sans doute en partie similaires à ce que l'on retrouve dans d'autres sociétés. Comme en France, le jeu semble répondre au besoin de contrôle sur son destin social et une volonté de prendre des risques à la façon d'un entrepreneur (Amadieu, 2015). La valorisation sociale de la prise de risque avec l'argent est forte en Chine et plus particulièrement associée au rôle masculin. De plus, le jeu d'argent en Chine est une pratique ancienne (loteries pour le financement de la grande muraille, bien avant les cas similaires rapportés en occident), très répandue et socialement largement acceptée. De nombreux jeux ont été inventés (Keno, Mah Jong) et l'omniprésence du jeu dans le plus petit village, son rôle crucial dans la " chaleur sociale " (social heat) a bien été mis en lumière par les anthropologues (Steinmuller, 2011).

Le jeu avec de petites sommes d'argent et lorsque le joueur n'est pas animé du désir de gagner de l'argent est considéré comme anodin («gaming»). Dans la culture chinoise, « miser petit est amusant, mais miser à l'excès ruine l'esprit » Le jeu avec de petites mises est acceptable et considéré comme un jeu divertissant (" wán shua » ou " yóu xì ») plutôt que comme du jeu d'argent («du bo») (Hu, 2015; Wu et Lau, 2015, p. 210). La délimitation entre le jeu modéré d'une part et le jeu excessif d'autre part est ancrée dans la culture populaire chinoise. La partie de cartes reste une activité familiale ou amicale où l'argent n'est présent que pour intéresser la partie. Selon Chen Haiping, chercheur au centre de recherche sur la loterie de l'Université Normale de Pékin, ce qui rend le jeu illégal, c'est le fait de jouer avec pour motivation de gagner de l'argent, le jeu sans volonté de profit est légal ${ }^{6}$.

41 Parce que le jeu fait partie intégrante d'une culture, qu'il est régulé par des normes et des rites, limité dans l'espace du cercle familial et dans le temps des célébrations, il entre pour une part majoritaire dans la catégorie du « jeu social» (Clarke et al., 2006). Le jeu (" gaming ») en Chine a une fonction sociale très importante, étant vu comme une opportunité pour construire des liens sociaux et les enfants sont encouragés à apprendre à jouer en famille (Steinmuller, 2011). L'argent peut en être absent, mais le plus souvent il est présent. Jouer en famille ou entre amis est généralement envisagé 
sous un jour favorable, sauf pour celui qui joue trop, qui perd plus qu'il ne devrait, qui masque ses pertes à son entourage ou délaisse son foyer pour passer ses nuits au jeu. Celui-ci sera alors perçu comme irresponsable, victime d'un vice très répandu, comme la prostitution ou la criminalité. La déviance est liée à de mauvaises influences et il n'y a pas de circonstances atténuantes, en particulier pas d'idée de maladie mentale qui atténuerait la responsabilité du joueur : « les personnes qui jouent de façon immodérée et ruinent leur vie sont considérées comme mauvaises plutôt que folles " (Lee, 1996, cité par Papineau, 2013). La rectification passe par la punition et la rééducation, pas par le traitement médical.

\section{Des réticences populaires au processus de médicalisation}

Si la médicalisation du jeu ne s'opère pas, c'est aussi en raison des réticences culturelles du public à la médicalisation. Le traitement des addictions, et plus particulièrement des addictions comportementales, fait l'objet de débats intenses et réguliers en Chine (Bax, 2016). Plusieurs cliniques, nous l'avons vu, ont été accusées de mauvais traitements sur les patients, souvent des adolescents, les conséquences étant parfois fatales. ${ }^{7}$ Cela pourrait conduire à regarder avec suspicion les traitements cliniques des addictions comportementales.

Il existe par ailleurs des réticences au sein du public liées au refus de l'étiquetage de la déviance en un sens médical. Un joueur ayant des problèmes de jeu le niera ou bien cherchera à éviter la honte et l'embarras public. Demander de l'aide extérieure serait la preuve d'un échec familial, puisque le problème devrait être maintenu dans l'intimité de la famille. Pour ne pas "perdre la face ", il est généralement préférable de donner l'apparence d'un fonctionnement normal de la famille (Fong \& Tsuang, 2007). Le traitement médical des troubles liés au jeu est perçu comme stigmatisant (Radermacher et al., 2017), beaucoup de Chinois percevant le fait d'avoir un désordre d'ordre mental comme un signe de faiblesse. Le joueur en difficulté aura alors plutôt tendance à se cacher aux yeux des autres qu'à chercher de l'aide auprès de professionnels (Loo, Raylu \& Oei, 2008). Mais au-delà de ces obstacles culturels, les contraintes sont aussi institutionnelles, l'offre de soins étant tout simplement insuffisante.

Toutefois le langage de l'addiction n'est pas absent, mais sous une forme différente. Des paniques morales liées aux "épidémies » d'addiction comportementales (Szablewicz, 2010), notamment aux jeux vidéo ou à internet surgissent régulièrement dans les médias officiels et donnent lieu à des efforts d'encadrement par crainte du développement d'une nouvelle forme d'« opium de l'esprit » (Golub \& Lingley, 2008). Le «malade du jeu » - que ce soit avec ou sans argent - doit être traité, moins parce qu'il se met en danger ou est en souffrance, mais parce qu'il s'écarte du droit chemin et n'assume plus ses responsabilités de fils, de mari ou de père. Il ne s'agit alors pas d'assistance, mais plutôt d'un encadrement et d'une rectification ayant pour but de protéger la famille et la société dans son ensemble. En affaissant la moralité des individus, l'addiction menace la société, d'où le parallèle fait avec l'opium au temps de la dynastie de Qing (Golub \& Lingley, 2008). 


\section{En France une diffusion contrastée auprès du grand public et des joueurs du modèle de l'addiction au jeu}

leurs comportements présentant le bénéfice moral de la déculpabilisation. Mais ce qui ressort de l'analyse des discours de joueurs français rencontrant des problèmes de contrôle sur leur pratique de jeu est une métaphore du jeu comme drogue, les opérateurs de jeu étant comparés à des dealers tirant profit de leur propre faiblesse de volonté (Amadieu, 2013). Les échecs et les dettes, les souffrances associées au jeu sont pour partie imputés à la façon dont les jeux eux-mêmes sont conçus. Les opérateurs de jeu, et singulièrement la FDJ en tant qu'elle est associée à l'État, sont perçus avec défiance. Il s'agit alors de représentations finalement opposées au modèle du jeu responsable qui tend à déresponsabiliser les opérateurs. La représentation du jeu assimilée à une drogue telle qu'elle est mobilisée par certains joueurs est une forme de réinterprétation du modèle médical, parfois très éloignée du discours scientifique même si elle se rapproche des représentations de l'addiction au jeu véhiculées par certains groupes d'entraide (type Joueurs anonymes).

\section{Conclusion}

Le modèle de l'addiction au jeu repose sur un processus de médicalisation née de progrès scientifiques qui rencontre un écho du fait de sa mobilisation possible par les pouvoirs publics et les opérateurs. Il se présente comme une sorte de modèle intellectuel « prêt à l'usage » qui permet avantageusement de légitimer un marché à la légitimité contestée. Mais ce processus n'est pas adopté de façon universelle car il peut 
se voir offrir des résistances politiques et axiologiques qui tendent à nuancer l'idée d'une convergence des modes de gouvernance du jeu. C'est le cas en Chine où le contrôle du jeu ne se fait pas par la grammaire libérale du jeu responsable, mais par le truchement plus direct de la prohibition ou de l'euphémisation. En cela la gouvernance chinoise du jeu tend plutôt à se rapprocher du « modèle de l'alibi » décrit par Kingma (2008) dans sa typologie des régimes de gouvernance dans la mesure où le jeu n'y est toléré qu'en tant qu'il profite à des œuvres de charité et permet de réduire l'offre illégale.

Cette résistance procède en partie seulement d'un «retard » selon les canons de la science et de l'administration de la preuve occidentale en matière de recherche sur les problèmes associés aux jeux d'argent, mais surtout d'une interprétation différentielle des résultats de la recherche qui privilégie une approche morale condamnant le jeu. On ne peut préjuger de l'avenir, mais il semblerait que la tendance soit à un renforcement des contrôles et à une réaffirmation des particularités chinoises, en même temps qu'un retour à une forme de pureté communiste à travers la lutte contre les vices importés tels que les jeux vidéo, les drogues ou les jeux en ligne. Là où l'État français tend à ne pas restreindre les consommations jugées majoritairement inoffensives, l'État chinois se situe à un niveau plus moral et souhaite promouvoir la vertu. Il n'est pas exclu non plus que la prohibition du jeu fasse école, dans la mesure où une majorité de la population mondiale vit d'ores et déjà dans des pays prohibitionnistes.

Une comparaison avec le traitement réservé aux jeux vidéo souligne en creux la spécificité des jeux d'argent dans le contexte chinois. En effet, les jeux vidéo en Chine font l'objet d'une médicalisation avancée, tout à fait à l'inverse des jeux d'argent. Plusieurs méta-analyses, en particulier auprès des populations de jeunes et d'étudiants, ont déjà été réalisées. Parmi les plus conséquentes, citons une étude de prévalence à partir de 36 études en Chine, cumulant 362328 joueurs sur internet (Long et al., 2018) ou encore une autre regroupant 70 études et 122454 étudiants d'université (Li et al., 2018). Ces effectifs impressionnants témoignent d'une grande préoccupation pour la problématique de l'addiction aux jeux vidéo et à internet chez les jeunes, en particulier lorsqu'ils sont encore en études. Comparativement aux jeux d'argent, cela correspond à un effort de recherche supérieur, alors même que les débats scientifiques internationaux sont plus vifs concernant la validité de la catégorie.

51 L'inquiétude pour les usages addictifs chez les adolescents est particulièrement vive, sans doute car les taux de prévalence identifiés du jeu en ligne problématique sont sans commune mesure avec ce que l'on trouve dans d'autres pays, aux USA et en Europe : entre 3,5\% et $17 \%$ selon les études, contre autour de $1 \%$ généralement (Long et al., 2018). Peut-être également en raison du fait que le phénomène touche prioritairement des jeunes scolarisés ou en étude, dans un contexte culturel où la réussite scolaire est cruciale. Ces constats scientifiques ont conduit à des mesures de limitation drastique de l'offre de jeu: pression intense de la part du gouvernement sur Tencent, le premier producteur de jeux vidéo au monde, afin d'interdire certains jeux, obligation de mettre en place la reconnaissance faciale et le contrôle de l'identité des joueurs en lien avec le fichier de la police. En France, si la médicalisation du jeu vidéo est en cours elle ne conduit pas à des mesures restrictives et d'encadrement du marché.

52 La pratique des jeux vidéo suit un processus de médicalisation, en particulier pour les adolescents, mais le choix en Chine se porte encore une fois sur une politique prohibitionniste plutôt qu'une autre version de jeu responsable. Ainsi l'existence d'une 
recherche importante en matière de jeu vidéo problématique ne s'assortit pas nécessairement de mesures de gouvernance de type libérale. Si en France la mobilisation des travaux scientifiques sur l'addiction au jeu est une condition essentielle à l'auto-régulation de l'industrie du jeu d'argent, le cas des jeux vidéo suggère qu'il n'existe pas de lien nécessaire entre médicalisation et mode de gouvernance libéral.

\section{BIBLIOGRAPHIE}

AMADIEU T. (2013), Sociologie des jeux de hasard. Croyances et rationalité, Thèse de doctorat, Université Paris-Sorbonne.

AMADIEU T. (2015), « Prises de risques délibérées avec l'argent : les modalités de consommation des jeux de hasard », Revue française de sociologie, 56(4), pp. 643-672.

AMERICAN PSYCHIATRIC ASSOCIATION (2013), Diagnostic and statistical manual of mental disorders (5th ed.), Washington, DC: Author.

BAX T. (2016), « "Internet gaming disorder” in China: Biomedical sickness or sociological badness? ", Games and Culture, 11(3), pp. 233-255.

BECKER H. S. (1963). Outsiders: Studies in the Sociology of Deviance, New York, The Free Press.

BLASZCZYNSKI A., LADOUCEUR R., \& SHAFFER H. J. (2004). « A science-based framework for responsible gambling: The Reno model », Journal of Gambling studies, 20(3), pp. 301-317.

BRODY A. (2015), « Pour une approche du gambling en termes de jeu », Sciences du jeu, $n^{\circ} 3$, [https://journals.openedition.org/sdj/465 ?lang =fr].

CHINESE SOCIETY OF PSYCHIATRY, (2001), Chinese Classification of Mental Disorders, $3^{\text {rd }}$ ed, Beijing, China.

CAMPBELL C. S., \& SMITH G. J. (2003), « Gambling in Canada-From vice to disease to responsibility: A negotiated history », Canadian Bulletin of Medical History, 20(1), pp. 121-149.

CASSIDY R. (2014), « Fair game? Producing and publishing gambling research », International Gambling Studies, 14(3), pp. 345-353.

CASSIDY R., LOUSSOUARN C., \& PISAC A. (2013). Fair game: Producing gambling research, Goldsmiths, University of London.

CHUI W. Y., LEE S. K., MOK Y. L., \& TSANG C. K. (2018), « The Diagnostic Criteria of Gambling Disorder of DSM-5 in Chinese Culture: By Confirmatory Factor Analysis (CFA) and Item Response Theory (IRT) », in M.-T. Leung, L.-M. Tan (eds.), Applied Psychology Readings, Springer, Singapore, pp. 73-86.

CONRAD P. (1992), « Medicalization and social control », Annual Review of Sociology, 18(1), pp. 209-232.

CONRAD P. (2005), « The shifting engines of medicalization », Journal of health and social behavior, 46(1), pp. 3-14. 
CONRAD P., \& SCHNEIDER J. W. (2010), Deviance and medicalization: From badness to sickness, Philadelphie, Temple University Press [1980].

CLARKE, D., TSE, S., ABBOTT, M., TOWNSEND, S., KINGI, P., \& MANAIA, W. (2006), « Key indicators of the transition from social to problem gambling ", International Journal of Mental Health and Addiction, 4(3), pp. 247-264.

DEMEULENAERE P. (2015), Homo œeconomicus : enquête sur la constitution d'un paradigme, Paris, PUF.

DOWLING N. A., MERKOURIS S. S., GREENWOOD C. J., OLDENHOF E., TOUMBOUROU J. W., \& YOUSSEF G. J. (2017), « Early risk and protective factors for problem gambling: A systematic review and meta-analysis of longitudinal studies ", Clinical psychology review, 51, pp. 109-124.

DUROY Q., \& WISMAN J. D. (2017), « Le monopole de l'État français sur les jeux d'argent : de l'art d'extorquer des fonds aux plus démunis ", Revue de la régulation. Capitalisme, institutions, pouvoirs, 22, https://journals.openedition.org/regulation/12532.

FONG T. W., \& TSUANG J. (2007), « Asian-Americans, addictions, and barriers to treatment », Psychiatry (Edgmont), 4(11), pp. 51-59.

GIESLER M. \& VERESIU E. (2014), « Creating the responsible consumer : Moralistic governance regimes and consumer subjectivity », Journal of Consumer Research, 41(3), pp. 840-857.

GOLUB A., \& LINGLEY K. (2008), « "Just like the qing empire” internet addiction, MMOGs, and moral crisis in contemporary China ", Games and Culture, 3(1), pp. 59-75.

HAIDT J. (2012), The righteous mind : Why good people are divided by politics and religion, New York, Vintage.

HANCOCK L. \& HAO Z., (2016), « Gambling regulatory regimes and the framing of "responsible gambling" by transnational casino corporations : Asia-Pacific regimes in comparative perspective », Asia Pacific Journal of Public Administration, 38(3), pp. 139-153.

HANCOCK L., \& SMITH G. (2017), « Critiquing the Reno Model I-IV international influence on regulators and governments (2004-2015)-the distorted reality of "responsible gambling" ", International Journal of Mental Health and Addiction, 15 (6), pp. 1151-1176.

HARRIS A., \& GRIFFITHS M. D. (2017), « A critical review of the harm-minimisation tools available for electronic gambling ", Journal of Gambling Studies, 33(1), pp. 187-221.

HING N., RUSSELL A. M. T., \& HRONIS A. (2018), « A definition and set of principles for responsible consumption of gambling ", International Gambling Studies, 18(3) pp. 359-382

HUANG X., SHIJUN D., \& GEORGE S. (2014), « Gambling addiction in China : a survey of Chinese psychiatrists », International Psychiatry, 11(4), pp. 98-99.

HUNT C. J., \& BLASZCZYNSKI A. (2019), « Gambling disorder as a clinical phenomenon ». In A. Heinz, N. Romanczuk-Seiferth, M. N. Potenza (eds.), Gambling Disorder, Springer, Cham, pp. 15-27.

HU S. (2015). La loterie en Chine : Etat-croupier et joueurs-coolies. Jeux de hasard et mutations sociétales, Paris, L'Harmattan.

HU S. (2015) ; « Entre “jouer avec l'argent” et "parier pour" l'argent : une analyse sociologique du double statut de l'argent du jeu en Chine », Sciences du jeu, $n^{\circ} 3$ https://journals.openedition.org/ $\operatorname{sdj} / 415$.

JODELET D. (2003), « Représentations sociales : un domaine en expansion. », in D. Jodelet (dir.), Les représentations sociales, Paris, PUF, pp. 45-78. 
KINGMA S. F. (2008), « The liberalization and (re) regulation of Dutch gambling markets : National consequences of the changing European context », Regulation \& Governance, 2(4), pp. 445-458.

KOSTKA G. (2019), « China's social credit systems and public opinion : Explaining high levels of approval », New media \& society, 21(7), pp. 1565-1593.

LEE S. (2001), « From diversity to unity : the classification of mental disorders in 21st-century China », Psychiatric Clinics of North America, 24(3), pp. 421-431.

LI L., XU D. D., CHAI J. X., WANG D., LI L., ZHANG L., \& XIANG Y. T. (2018), « Prevalence of Internet addiction disorder in Chinese university students : A comprehensive meta-analysis of observational studies ", Journal of behavioral addictions, 7(3), pp. 610-623.

LIU K. (2009) « Pathological gambling and strategy research on pathological gambling of lottery buyers ». Journal of Shandong Institute of Physical Education and Sports, 25, pp. 6-10.

LIU K. (2010), « Research on pathological gambling behavior during Chinese sports lottery's development », Sports Cult Guide, 10, pp. 69-73.

LIVINGSTONE C., \& ADAMS P. J. (2016), « Clear principles are needed for integrity in gambling research ", Addiction, 111(1), pp. 5-10.

LONG J., LIU T., LIU Y., HAO W., MAURAGE P., \& BILLIEUX J. (2018), « Prevalence and correlates of problematic online gaming : a systematic review of the evidence published in Chinese ", Current Addiction Reports, 5(3), pp. 359-371.

LOO J. M., RAYLU N., \& OEI T. P. S. (2008), « Gambling among the Chinese : A comprehensive review ", Clinical psychology review, 28(7), pp. 1152-1166.

MANGEL A.-C. \& TRESPEUCH M. (2009), « La RSE et les jeux d'argent : les nouveaux enjeux de la régulation », Sociologies pratiques, $\mathrm{n}^{\circ}$ 18, pp. 91-107.

MARIONNEAU V., \& JÄRVINEN-TASSOPOULOS J. (2017), « Consumer protection in licensed online gambling markets in France : the role of responsible gambling tools ", Addiction Research \& Theory, 25(6), pp. 436-443.

MONAGHAN S., \& BLASZCZYNSKI A. (2010), « Impact of mode of display and message content of responsible gambling signs for electronic gaming machines on regular gamblers ", Journal of Gambling Studies, 26(1), pp. 67-88.

OEI T. P., LIN J. \& RAYLU N. (2007) « Validation of the Chinese version of the Gambling Related Cognitions Scale (GRCS-C) », Journal of Gambling Studies, 23(3), pp. 309-322.

OEI T. P. S., LIN J. \& RAYLU N. (2007), « Validation of the Chinese Version of the Gambling Urges Scale (GUS-C) », International Gambling Studies, 7(1), pp. 101-111.

PAPINEAU E. (2005), « Pathological gambling in Montreal's Chinese community : an anthropological perspective ", Journal of Gambling Studies, n² 21, pp. 157-78.

PAULES X. (2016), «Ce que les statistiques ne disent pas : la réinvention de l'offre des jeux de casino à Macao depuis 2002 ", Sciences du jeu, n 5 https://journals.openedition.org/sdj/562.

RADERMACHER H., DICKINS M., ANDERSON C. \& FELDMAN S. (2017), « Perceptions of gambling in Tamil and Chinese communities in Australia : The role of saving face in perpetuating gambling stigma and hindering help seeking », Journal of Gambling Issues, $n^{\circ} 34$, pp. 77-99.

RAMADAN Z. (2018), " The gamification of trust : the case of China's "social credit" ", Marketing Intelligence \& Planning, 36(1), pp. 93-107. 
REITH G. (2007), « Gambling and the contradictions of consumption : A genealogy of the "pathological" subject », American behavioral scientist, 51(1), pp. 33-55.

ROSECRANCE J., (1985), « Compulsive gambling and the medicalization of deviance », Social problems, 32(3), pp. 275-284.

ROSSOL J. (2001), « The medicalization of deviance as an interactive achievement : The construction of compulsive gambling ", Symbolic Interaction, 24(3), pp. 315-341.

SZABLEWICZ M. (2010), « The ill effects of "opium for the spirit" : a critical cultural analysis of China's Internet addiction moral panic », Chinese Journal of Communication, 3(4), pp. 453-470.

STEINMULLER H. (2011), " The moving boundaries of social heat : gambling in rural China », Journal of Royal Anthropolical Institute, ${ }^{\circ}$ 17, pp. 263-280.

SUISSA J. A. (2005), « La construction d'un problème social en pathologie : le cas des jeux de hasard et d'argent (gambling)», Nouvelles pratiques sociales, 18(1), pp. 148-161.

TANG C. S. K., \& WU, A. M. S. (2009). Screening for college problem gambling in Chinese societies : Psychometric properties of the Chinese version of the South Oaks Gambling Screen (C-SOGS), International Gambling Studies, 9(3), pp. 263-274.

TANG C. S. K., WU, A. M. S., TANG, J. Y. C., \& YAN, E. C. W. (2010). Reliability, validity, and cut scores of the South Oaks Gambling Screen (SOGS) for Chinese, Journal of Gambling Studies, 26(1), pp. $145-158$.

TAO V. Y. K., WU A. M. S., CHEUNG S. F., \& TONG K. K. (2011). « Development of an indigenous inventory GMAB (Gambling Motives, Attitudes and Behaviors) for Chinese gamblers : An exploratory study ", Journal of Gambling Studies, $\mathrm{n}^{\circ}$ 27, pp. 99-113.

TONG K. K., HUNG E. P., LEI C. M., \& WU A. M. (2018), « Public awareness and practice of responsible gambling in Macao ", Journal of gambling studies, 34(4), pp. 1261-1280.

WANG P. \& ANTONOPOULOS G.A., (2016), « Organized crime and illegal gambling : How do illegal gambling enterprises respond to the challenges posed by their illegality in China? ", Australian \& New Zealand Journal of Criminology, 49(2), pp. 258-280.

WOOD R. T., \& WOHL M. J. (2015), « Assessing the effectiveness of a responsible gambling behavioural feedback tool for reducing the gambling expenditure of at-risk players ", International Gambling Studies, 15(2), pp. 1-16.

WORLD HEALTH ORGANIZATION (2018), International classification of diseases for mortality and morbidity statistics ( $11^{\text {th }}$ Revision).

WU A. M., LAI M. H., TONG K. K., \& TAO V. Y. (2013), « Chinese attitudes, norms, behavioral control and gambling involvement in Macao », Journal of Gambling Studies, 29(4), pp. 749-763.

WU A. M. \& LAU J. T., (2015), « Gambling in China : Socio-historical evolution and current challenges ", Addiction, 110(2), pp. 210-216.

WU A. M. \& TANG C. S., (2011), « Validation of the Chinese version of the gambling motivation scale (C-GMS) », Journal of Gambling Studies, 27(4), pp. 709-724.

ZOU Y. Z., CUI J. F., HAN B., MA A. L., LI M. Y., \& FAN H. Z. (2008), « Chinese psychiatrists views on global features of CCMD-III, ICD-10 and DSM-IV », Asian Journal of Psychiatry, 1(2), pp. 56-59. 


\section{NOTES}

1. Le Qigong est une pratique traditionnelle chinoise associant méditation, respiration et coordination des mouvements et des postures. Elle fait l'objet d'une attention particulière des autorités chinoises en raison de son association avec le mouvement du Falun Gong considéré comme un « culte diabolique ».

2. Le diagnostic chinois est utilisé dans plusieurs enquêtes: Tang \& Wu (2009). Et l'alternative étrangère a été étudiée de façon psychométrique pour évaluer sa pertinence (Tang et al., 2010).

3. https://www.caixinglobal.com/2020-01-06/chinas-lotteries-look-like-bad-bets-as-supervisiontightens-101501515.html, consulté le 04/06/2020.

4. Notamment une petite bannière rouge en bas de page indiquant « Fixez vos limites quand vous jouez à la loterie, misez de façon raisonnable !».

5. https://web.archive.org/web/20090420061045/http://www.sd.xinhuanet.com/sdsq/ 2009-03/13/content_15941408.htm consulté le 04/06/2020.

6. https://foreignpolicy.com/2013/09/03/the-big-bet/, consulté le 15/10/2010.

7. https://www.bbc.com/news/world-asia-china-40920488, consulté le 06/06/2020.

\section{RÉSUMÉS}

Cet article propose une comparaison entre deux régimes de représentation contrastés: le paradigme individualiste français et le paradigme moraliste chinois. Celle-ci permet de mieux saisir les conditions de diffusion du discours scientifique du trouble lié au jeu et les transformations des différents régimes de gouvernance du risque associé aux jeux. Il s'agit de comprendre comment les discours savants et les efforts de régulations peuvent interagir avec les représentations ordinaires; là où le discours médical en France offre pour partie une déresponsabilisation, en Chine c'est une condamnation morale qui domine en tant que le joueur mettrait en danger son entourage, sa famille et plus largement l'harmonie sociale. À partir d'une analyse des différents niveaux de représentations relatives aux jeux d'argent - représentations savantes, opérationnelles et ordinaires - cette étude explore l'hypothèse selon laquelle la diffusion du modèle du jeu responsable repose sur l'adhésion d'un ensemble d'acteurs à des représentations du monde libérales qui accompagnent le développement d'une logique de marché, mais qui ne sont pas nécessairement uniformément adoptées.

This article offers a comparative study of two contrasting schemes of representation: the French individualist paradigm and the Chinese moralist paradigm. It provides a better understanding of the conditions of diffusion of the scientific discourse of gambling disorders and the transformations of the various regimes of governance of the risk associated with gambling. The aim is to understand how scholarly discourse and regulatory efforts can interact with ordinary representations; where medical discourse in France partly offers a disempowerment, in China it is a moral condemnation that dominates as the gambler endangers his entourage, his family and more broadly social harmony. Based on an analysis of the different levels of representations relating to gambling - academic, operational and ordinary representations - this study explores the hypothesis that the spread of the responsible gambling model is based on various actors adhering to liberal world representations that accompany the development of a rationale of free market but are not necessarily uniformly adopted. 
INDEX

Mots-clés : jeu d'argent, addiction, représentations sociales, jeu responsable, médicalisation

Keywords : gambling, addiction, social representations, responsible gambling, medicalization

\section{AUTEUR}

\section{THOMAS AMADIEU}

ESSCA (campus de Shanghai, Chine) / GEMASS (Sorbonne Université/CNRS) 